\title{
$p$-Capitulation over Number Fields with p-Class Rank Two
}

\author{
Daniel C. Mayer \\ Graz, Austria \\ Email: algebraic.number.theory@algebra.at \\ Received 12 May 2016; accepted 12 July 2016; published 15 July 2016
}

\section{Abstract}

Theoretical foundations of a new algorithm for determining the $p$-capitulation type $\varkappa(K)$ of a number field $K$ with $p$-class rank $\varrho=2$ are presented. Since $\varkappa(K)$ alone is insufficient for identifying the second $p$-class group $\mathfrak{G}=\operatorname{Gal}\left(\mathrm{F}_{p}^{2} K \mid K\right)$ of $K$, complementary techniques are developed for finding the nilpotency class and coclass of $\mathfrak{G}$. An implementation of the complete algorithm in the computational algebra system Magma is employed for calculating the Artin pattern $\operatorname{AP}(K)=(\tau(K), \varkappa(K))$ of all 34631 real quadratic fields $K=\mathbb{Q}(\sqrt{d})$ with discriminants $0<d<10^{8}$ and 3 -class group of type $(3,3)$. The results admit extensive statistics of the second 3-class groups $\mathfrak{G}=\operatorname{Gal}\left(F_{3}^{2} K \mid K\right)$ and the 3-class field tower groups $G=\operatorname{Gal}\left(F_{3}^{\infty} K \mid K\right)$.

\section{Keywords}

Hilbert $p$-Class Field Tower, Maximal Unramified Pro- $p$ Extension, $p$-Capitulation of Class Groups, Real Quadratic Fields $(3,3)$

\section{Introduction}

Let $p$ be a prime number. Suppose that $K$ is an algebraic number field with $p$-class group $\mathrm{Cl}_{p} K:=\mathrm{Syl}_{p} \mathrm{Cl}_{K}$ and p-elementary class group $\mathrm{E}_{p} K:=\mathrm{Cl}_{p} K \bigotimes_{\mathbb{Z}_{p}} \mathbb{F}_{p}$. By class field theory ([1] Cor. 3.1, p. 838), there exist precisely $n:=\frac{p^{\varrho}-1}{p-1}$ distinct (but not necessarily non-isomorphic) unramified cyclic extensions $L_{i} \mid K, 1 \leq i \leq n$, of degree $p$, if $K$ possesses the $p$-class rank $\varrho:=\operatorname{dim}_{\mathbb{F}_{p}} \mathrm{E}_{p} K$. For each $1 \leq i \leq n$, let $j_{L_{i} K}: \mathrm{Cl}_{p} K \rightarrow \mathrm{Cl}_{p} L_{i}$ denote the class extension homomorphism induced by the ideal extension monomorphism ([2] 1, p. 74). We let $U_{K}$, resp. $U_{L_{i}}$, be the group of units of $K$, resp. $L_{i}$.

Proposition 1.1. (Order of $\operatorname{ker} j_{L_{i} \mid K}$ )

The kernel ker $j_{L_{i} K}$ of the class extension homomorphism associated with an unramified cyclic extension $L_{i} \mid K$ of degree $L_{i}: K=p$ is a subgroup of the $p$-elementary class group $\mathrm{E}_{p} K$ and has the $\mathbb{F}_{p}$-dimension

$$
1 \leq \operatorname{dim}_{\mathbb{F}_{p}} \operatorname{ker} j_{L_{i} \mid K}=\log _{p}\left(L_{i}: K \cdot\left(U_{K}: \operatorname{Norm}_{L_{i} \mid K} U_{L_{i}}\right)\right) \leq \varrho .
$$


Proof. The proof of the inclusion ker $j_{L_{i} K} \leq \mathrm{E}_{p} K$ was given in ([2] 1, p. 74) for $p=3$, and generally in ([3] Prop. 4.3.(1), p. 484). The relation \# ker $j_{L_{i} \mid K}=L_{i}: K \cdot\left(U_{K}: \operatorname{Norm}_{L_{i} K} U_{L_{i}}\right)$ for the unramified extension $L_{i} \mid K$ is equivalent to the Theorem on the Herbrand quotient ([4] Thm. 3, p. 92) and was proved in [[3] Prop. 4.3, pp. 484-485]. According to Hilbert's Theorem 94 ([5] p. 279), the kernel ker $j_{L_{i} K}$ cannot be trivial.

Definition 1.1. For each $1 \leq i \leq n$, the elementary abelian p-group ker $_{L_{i} K}$ is called the p-capitulation kernel of $L_{i} \mid K$. We speak about total capitulation [6] [7] if $\operatorname{dim}_{\mathbb{F}_{p}} \operatorname{ker} j_{L_{i} \mid K}=\varrho$, and partial capitulation if $1 \leq \operatorname{dim}_{\mathbb{F}_{p}} \operatorname{ker} j_{L_{i} \mid K}<\varrho$.

If $p \geq 3$ is an odd prime, and $K=\mathbb{Q}(\sqrt{d})$ is a quadratic field with fundamental discriminant $d:=d_{K}$ and $p$-class rank $\varrho \geq 1$, then there arise the following possibilities for the $p$-capitulation kernel in any of the unramified cyclic relative extensions $L_{i} \mid K$ of degree $p$, which are absolutely dihedral extensions $L_{i} \mid \mathbb{Q}$ of degree $2 p$, according to ([3] Prop. 4.1, p. 482).

Corollary 1.1. (Partial and total p-capitulation over $K=\mathbb{Q}(\sqrt{d})$ with $\varrho \geq 2$ )

$$
\operatorname{dim}_{\mathbb{F}_{p}} \operatorname{ker} j_{L_{i} \mid K}= \begin{cases}1 & \text { if } K \text { is complex, } d<0, \\ 1 & \text { if } K \text { is real, } d>0 \text {, and } L_{i} \text { is of type } \delta, \\ 2 & \text { if } K \text { is real, } d>0 \text {, and } L_{i} \text { is of type } \alpha .\end{cases}
$$

The p-capitulation over $K$ is total if and only if $K$ is real with $\varrho=2$, and $L_{i}$ is of type $\alpha$.

Proof. In this special case of a quadratic base field $K$, the extensions $L_{i} \mid K, 1 \leq i \leq n$, are pairwise nonisomorphic although they share a common discriminant which is the pth power $d_{L_{i}}=d_{K}^{p}$ of the fundamental discriminant of $K$ [[1] Abstract, p. 831]. If $K$ is complex, the unit norm index equals 1 , since the cyclotomic quadratic fields do not possess unramified cyclic extensions of odd prime degree. If $K$ is real, we have $\left(U_{K}: \operatorname{Norm}_{L_{i} \mid K} U_{L_{i}}\right)=1 \Leftrightarrow L_{i}$ is of type $\delta$, and $\left(U_{K}: \operatorname{Norm}_{L_{i} \mid K} U_{L_{i}}\right)=p \Leftrightarrow L_{i}$ is of type $\alpha$ [[3] Prop. 4.2, pp. 482-483].

The organization of this article is the following. In §2, basic theoretical prerequisites for the new capitulation algorithm are developed. The implementation in Magma [8] consists of a sequence of computational techniques whose actual code is given in $\S 3$. The final $\S 4$ demonstrates the results of an impressive application to the case $p=3$, presenting statistics of all 3-capitulation types $\varkappa(K)$, Artin patterns $\mathrm{AP}(K)$, and second 3-class groups $\mathfrak{G}=\operatorname{Gal}\left(\mathrm{F}_{3}^{2} K \mid K\right)$ of the 34631 real quadratic fields $K=\mathbb{Q}(\sqrt{d})$ with discriminants $0<d<10^{8}$ and 3-class group of type $(3,3)$, which beats our own records in [3] §6 and [9] §6. Theorems concerning 3-tower groups $G=\operatorname{Gal}\left(\mathrm{F}_{3}^{\infty} K \mid K\right)$ with derived length $2 \leq \mathrm{dl}(G) \leq 3$ perfect the current state of the art.

\section{Theoretical Prerequisites}

In this article, we consider algebraic number fields $K$ with $p$-class rank $\varrho=2$, for a given prime number $p$. As explained in $\S 1$, such a field $K$ has $n=p+1$ unramified cyclic extensions $L_{i}$ of relative degree $p$.

Definition 2.1. By the Artin pattern of $K$ we understand the pair consisting of the family $\tau(K)$ of the $p$-class groups of all extensions $L_{1}, \ldots, L_{n}$ as its first component (called the transfer target type) and the p-capitulation type $\varkappa(K)$ as its second component (called the transfer kernel type),

$$
\operatorname{AP}(K):=(\tau(K), \varkappa(K)), \quad \tau(K):=\left(\mathrm{Cl}_{p} L_{i}\right)_{1 \leq i \leq n}, \quad \varkappa(K):=\left(\operatorname{ker} j_{L_{i} \mid K}\right)_{1 \leq i \leq n} .
$$

Remark 2.1. We usually replace the group objects in the family $\tau(K)$, resp. $\varkappa(K)$, by ordered abelian type invariants, resp. ordered numerical identifiers ([10] Rmk. 2.1).

We know from Proposition 1.1 that each kernel $\operatorname{ker} j_{L_{i} \mid K}$ is a subgroup of the $p$-elementary class group $\mathrm{E}_{p} K$ of $K$. On the other hand, there exists a unique subgroup $S<\mathrm{Cl}_{K}$ of index $p$ such that $S=\operatorname{Norm}_{L_{i} \mid K} \mathrm{Cl}_{L_{i}}$, according to class field theory. Thus we must first get an overview of the connections between subgroups of index $p$ and subgroups of order $p$ of $\mathrm{Cl}_{K}$.

Lemma 2.1. Let $p$ be a prime and $A$ be a finite abelian group with positive p-rank and with Sylow p-subgroup $\operatorname{Syl}_{p} A$. Denote by $A_{0}$ the complement of $\operatorname{Syl}_{p} A$ such that $A \simeq A_{0} \times \operatorname{Syl}_{p} A$. Then, any subgroup $S<A$ of index $p$ is of the form $S \simeq A_{0} \times U$ with a subgroup $U<\operatorname{Syl}_{p} A$ of index $p$.

Proof. Any subgroup $S$ of $A \simeq A_{0} \times \operatorname{Syl}_{p} A$ is of the shape $S \simeq S_{0} \times U$ with $S_{0} \leq A_{0}$ and $U \leq \operatorname{Syl}_{p} A$. We have $p=(A: S)=\left(A_{0} \times \operatorname{Syl}_{p} A: S_{0} \times U\right)=\left(A_{0}: S_{0}\right) \cdot\left(\operatorname{Syl}_{p} A: U\right)$. Since $\left(A_{0}: S_{0}\right)$ is coprime to $p$, we conclude that $S_{0}=A_{0}$ and $\left(\operatorname{Syl}_{p} A: U\right)=p$. 
An application to the particular case $A=\mathrm{Cl}_{K}$ and $S=\operatorname{Norm}_{L_{i} \mid K} \mathrm{Cl}_{L_{i}}<\mathrm{Cl}_{K}$ shows that $S \simeq\left(\mathrm{Cl}_{K}\right)_{0} \times U$ with $U=\operatorname{Norm}_{L_{i} K} \mathrm{Cl}_{p} L_{i}<\mathrm{Cl}_{p} K$.

Three cases must be distinguished, according to the abelian type of the $p$-class group $\mathrm{Cl}_{p} K$. We first consider the general situation of a finite abelian group $A$ with type invariants $\left(a_{1}, \ldots, a_{n}\right)$ having $p$-rank $r_{p}(A)=2$, that is, $n \geq 2, p\left|a_{n}, p\right| a_{n-1}$, but $\operatorname{gcd}\left(p, a_{i}\right)=1$ for $i<n-1$. Then the Sylow $p$-subgroup $\operatorname{Syl}_{p} A$ of $A$ is of type $\left(p^{u}, p^{v}\right)$ with integer exponents $u \geq v \geq 1$, and the $p$-elementary subgroup $A_{p}$ of $A$ is of type $(p, p)$. We select generators $x, y$ of $\operatorname{Syl}_{p} A=\langle x, y\rangle$ such that $\operatorname{ord}(x)=p^{u}$ and $\operatorname{ord}(y)=p^{v}$.

Lemma 2.2. Let $p$ be a prime number.

Suppose that $G$ is a group and $x \in G$ is an element with finite order $e:=\operatorname{ord}(x)$ divisible by $p$.

Then the power $x^{m}$ with exponent $m:=\frac{e}{p}$ is an element of order $\operatorname{ord}\left(x^{m}\right)=p$.

Proof. Generally, the order of a power $x^{m}$ with exponent $m \in \mathbb{Z}$ is given by

$$
\operatorname{ord}\left(x^{m}\right)=\frac{\operatorname{ord}(x)}{\operatorname{gcd}(m, \operatorname{ord}(x))} \text {. }
$$

This can be seen as follows. Let $d:=\operatorname{gcd}(m, e)$, and suppose that $m=d \cdot m_{0}$ and $e=d \cdot e_{0}$, then $\operatorname{gcd}\left(m_{0}, e_{0}\right)=1$. We have $\left(x^{m}\right)^{e_{0}}=x^{m_{0} \cdot d \cdot e_{0}}=\left(x^{e}\right)^{m_{0}}=1$, and thus $n:=\operatorname{ord}\left(x^{m}\right)$ is a divisor of $e_{0}$. On the other hand, $1=\left(x^{m}\right)^{n}=x^{m \cdot n}$, and thus $e=d \cdot e_{0}$ divides $m \cdot n=d \cdot m_{0} \cdot n$. Consequently, $e_{0}$ divides $m_{0} \cdot n$, and thus necessarily $e_{0}$ divides $n$, since $\operatorname{gcd}\left(m_{0}, e_{0}\right)=1$. This yields $n=e_{0}$, as claimed.

Finally, put $m:=\frac{e}{p}$, then $\operatorname{ord}\left(x^{m}\right)=\frac{e}{\operatorname{gcd}(m, e)}=\frac{e}{\operatorname{gcd}\left(\frac{e}{p}, e\right)}=\frac{e}{\frac{e}{p}}=p$.

Now, we apply Lemma 2.2 to the situation where $A$ is a finite abelian group with type invariants $\left(a_{1}, \ldots, a_{n}\right)$ having $p$-rank $r_{p}(A)=2$, that is, $n \geq 2, p\left|a_{n}, p\right| a_{n-1}$.

Proposition 2.1. (p-elementary subgroup)

If $A$ is generated by $g_{1}, \ldots, g_{n}$, then the $p$-elementary subgroup of $A$ is given by $\left\langle g_{n-1}^{a_{n-1} / p}, g_{n}^{a_{n} / p}\right\rangle$.

Proof. Let generators of $A$ corresponding to the abelian type invariants $\left(a_{1}, \ldots, a_{n}\right)$ be $\left(g_{1}, \ldots, g_{n}\right)$, in particular, the trailing two generators have orders $\operatorname{ord}\left(g_{n-1}\right)=a_{n-1}$ and $\operatorname{ord}\left(g_{n}\right)=a_{n}$ divisible by $p$. According to Lemma 2.2, the powers $g_{n-1}^{a_{n-1} / p}$ and $g_{n}^{a_{n} / p}$ have exact order $p$ and thus generate the $p$-elementary subgroup of $A$.

Proposition 2.2. (Subgroups of order $p$ )

If the $p$-elementary subgroup $A_{p}=\langle w, z\rangle$ of $A$ is generated by $w, z$, then the subgroups of $A_{p}$ of order $p$ can be given by $M_{1}=\langle z\rangle$ and $M_{i}=\left\langle w z^{i-2}\right\rangle$ for $2 \leq i \leq p+1$.

Proof. According to the assumptions, $A_{p}$ is elementary abelian of rank 2 , that is, of type $(p, p)$, and consists of the $p^{2}$ elements $\left\{w^{i} z^{j} \mid 0 \leq i, j \leq p-1\right\}$, in particular, $w^{0} z^{0}=1$ is the neutral element. A possible selection of generators for the $\frac{p^{2}-1}{p-1}=p+1$ cyclic subgroups $M_{i}$ of order $p$ is to take $M_{1}=\langle z\rangle$ and $M_{i}=\left\langle w z^{i-2}\right\rangle$ for $2 \leq i \leq p+1$, since the two cycles of powers of $w z^{i}$ and $w z^{j}$ for $1 \leq i<j \leq p-1$ meet in the neutral element only.

Proposition 2.3. (Connection between subgroups of index $p$, resp. order $p$ )

1) If $u=v=1$, which is equivalent to $A_{p}=\operatorname{Syl}_{p} A$, then

$$
\left\{U<\operatorname{Syl}_{p} A \mid\left(\operatorname{Syl}_{p} A: U\right)=p\right\}=\left\{U<A_{p} \mid \# U=p\right\} .
$$

2) If $u>v=1$, then there exists a unique bicyclic subgroup $\left\langle x^{p}, y\right\rangle$ of index $p$ which contains $A_{p-1}$ The other $p$ subgroups $U$ of index $p$ are cyclic of order $p^{u}$, and they only contain the unique subgroup $\left\langle x^{p^{p-1}}\right\rangle$ of $A_{p}$ generated by the $p^{u-1}$ th powers.

3) If $u \geq v>1$, then each subgroup $U<\operatorname{Syl}_{p} A$ of index $p$ completely contains the $p$-elementary subgroup $A_{p}$.

Proof. If $u=v=1$, then $\operatorname{Syl}_{p} A \simeq(p, p) \simeq A_{p}$. Thus, $p^{2}=\left(A_{p}: 1\right)=\left(A_{p}: U\right) \cdot(U: 1)$ implies

$\left(A_{p}: U\right)=(U: 1)=p$, for each proper subgroup $U$.

If $u>v=1$, then a subgroup $U$ of index $p$ is either of type $\left(p^{u}\right)$, i.e., cyclic, or of type $\left(p^{u-1}, p\right) \geq(p, p)$.

If $u \geq v>1$, then each subgroup $U$ of index $p$ is either of type $\left(p^{u}, p^{v-1}\right)>(p, p)$ or of type 
$\left(p^{u-1}, p^{v}\right)>(p, p)$.

Theorem 2.1. (Taussky's conditions A and B, see Formula (5))

Let $L \mid K$ be an unramified cyclic extension of prime degree $p$ of a base field $K$ with $p$-class rank $\varrho=2$. Suppose that $S=\operatorname{Norm}_{L \mid K} \mathrm{Cl}_{L}<\mathrm{Cl}_{K}$ and $U=\operatorname{Norm}_{L \mid K} \mathrm{Cl}_{p} L<\mathrm{Cl}_{p} K$ are the subgroups of index $p$ associated with $L \mid K$, according to class field theory.

Then, we generally have ker $j_{L \mid K} \cap S=\operatorname{ker} j_{L \mid K} \cap U$, and in particular:

1) If $u=v=1$, then

$L$ is of type A if either $\operatorname{ker} j_{L \mid K}=\mathrm{E}_{p} K$ or $\operatorname{ker} j_{L \mid K}=U$, and

$L$ is of type B if ker $j_{L \mid K} \notin\left\{\mathrm{E}_{p} K, U\right\}$.

2) If $u>v=1$, let $N:=\left\langle x^{p}, y\right\rangle\left\langle\mathrm{Cl}_{p} K\right.$ denote the unique bicyclic subgroup of index $p$, then

$L$ is of type A if either ker $j_{L \mid K}=\mathrm{E}_{p} K$ or $U=N$ or $U \neq N$ and $\operatorname{ker} j_{L \mid K}=\left\langle x^{p^{u-1}}\right\rangle$, and

$L$ is of type B if $U \neq N$ and $\operatorname{ker} j_{L \mid K} \notin\left\{\mathrm{E}_{p} K,\left\langle x^{p^{u-1}}\right\rangle\right\}$.

3) If $u \geq v>1$, then $L$ is always of type A.

Proof. This is an immediate consequence of Proposition 2.3.

Theorem 2.2. (Orbits of TKTs expressing the independence of renumeration)

1) If $u=v=1$, then $\varkappa \sim \lambda$ if and only if $\lambda=\sigma_{0}^{-1} \circ \varkappa \circ \sigma$ for some permutation $\sigma \in S_{p+1}$ and its extension $\sigma_{0} \in S_{p+2}$ with $\sigma_{0}(0)=0$.

2) If $u>v=1$, then $\varkappa \sim \lambda$ if and only if $\lambda=\pi_{0}^{-1} \circ \varkappa \circ \rho_{*}$ for two permutations $\pi, \rho \in S_{p}$ and the extensions $\pi_{0} \in S_{p+2}$ with $\pi_{0}(0)=0, \pi_{0}(p+1)=p+1$, and $\rho_{*} \in S_{p+1}$ with $\rho_{*}(p+1)=p+1$.

3) If $u \geq v>1$, then $\varkappa \sim \lambda$ if and only if $\lambda=\sigma_{0}^{-1} \circ \varkappa \circ \tau$ for two permutations $\sigma, \tau \in S_{p+1}$ and the extension $\sigma_{0} \in S_{p+2}$ with $\sigma_{0}(0)=0$.

Proof. The proof for the case $u=v=1$ was given in ([2] p. 79) and ([11] Rmk. 5.3, pp. 87-88). It is the unique case where subgroups of index $p$ coincide with subgroups of order $p$, and a renumeration of the former enforces a renumeration of the latter, expressed by a single permutation $\sigma \in S_{p+1}$ and its inverse $\sigma^{-1}$.

If $u>v=1$, then the distinguished subgroups $U_{p+1}=N=\left\langle x^{p}, y\right\rangle \simeq\left(p^{u-1}, p\right)$ of index $p$, and $V_{p+1}=\left\langle x^{p^{u-1}}\right\rangle$ of order $p$, should have the fixed subscript $p+1$. The other $p$ subgroups $U_{i}$, resp. $V_{i}$, can be renumerated completely independently of each other, which can be expressed by two independent permutations $\pi, \rho \in S_{p}$. For details, see ([11] Rmk. 5.6, p. 89).

In the case $u \geq v>1$, finally, the $p+1$ subgroups of index $p$ of $\mathrm{Cl}_{p} K$ and the $p+1$ subgroups of order $p$ of $\mathrm{Cl}_{p} K$ can be renumerated completely independently of each other, which can be expressed by two independent permutations $\sigma, \tau \in S_{p+1}$.

\section{Computational Techniques}

In this section, we present the implementation of our new algorithm for determining the Artin pattern $\operatorname{AP}(K)$ of a number field $K$ with $p$-class rank $\varrho=2$ in MAGMA [8] [12] [13], which requires version V2.21-8 or higher. Algorithm 3.1 returns the entire class group $C:=\mathrm{Cl}_{K}$ of the base field $K$, together with an invertible mapping $m C$ from classes to representative ideals.

Algorithm 3.1 (Construction of the base field $K$ and its class group $C$ )

Input: The fundamental discriminant $d$ of a quadratic field $K=\mathbb{Q}(\sqrt{d})$.

Code:

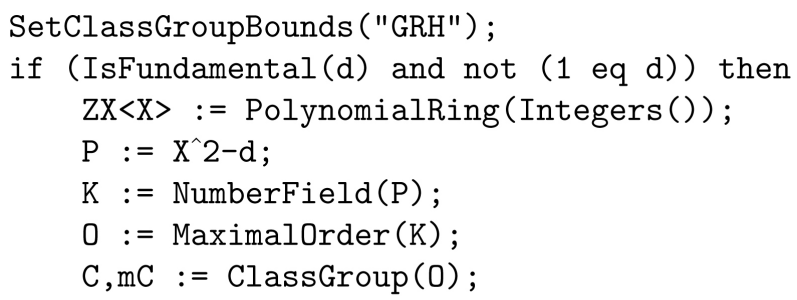

Output: The conditional class group $(C, m C)$ of the quadratic field $K$, assuming the GRH.

Remark 3.1. By using the statement $K$ : $=$ QuadraticField(d); the quadratic field $K=\mathbb{Q}(\sqrt{d})$ is constructed 
directly. However, the construction by means of a polynomial $P(X) \in \mathbb{Z} X$ executes faster and can easily be generalized to base fields $\mathrm{K}$ of higher degree.

For the next algorithm it is important to know that in the MAGMA computational algebra system [8], the composition $A \times A \rightarrow A,(x, y) \mapsto x+y$, of an abelian group $A$ is written additively, and abelian type invariants $\left(a_{1}, \ldots, a_{n}\right)$ of a finite abelian group $A$ are arranged in non-decreasing order $a_{1} \leq \ldots \leq a_{n}$.

Given the situation in Proposition 2.1, where $A$ is a finite abelian group having $p$-rank $r_{p}(A)=2$, Algorithm 3.2 defines a natural ordering on the subgroups $S$ of $A$ of index $(A: S)=p$ by means of Proposition 2.2, if the Sylow $p$-subgroup $\operatorname{Syl}_{p} A$ is of type $(p, p)$.

Algorithm 3.2. (Natural ordering of subgroups of index $p$ )

Input: A prime number $p$ and a finite abelian group $A$ with $p$-rank $r_{p}(A)=2$.

Code:

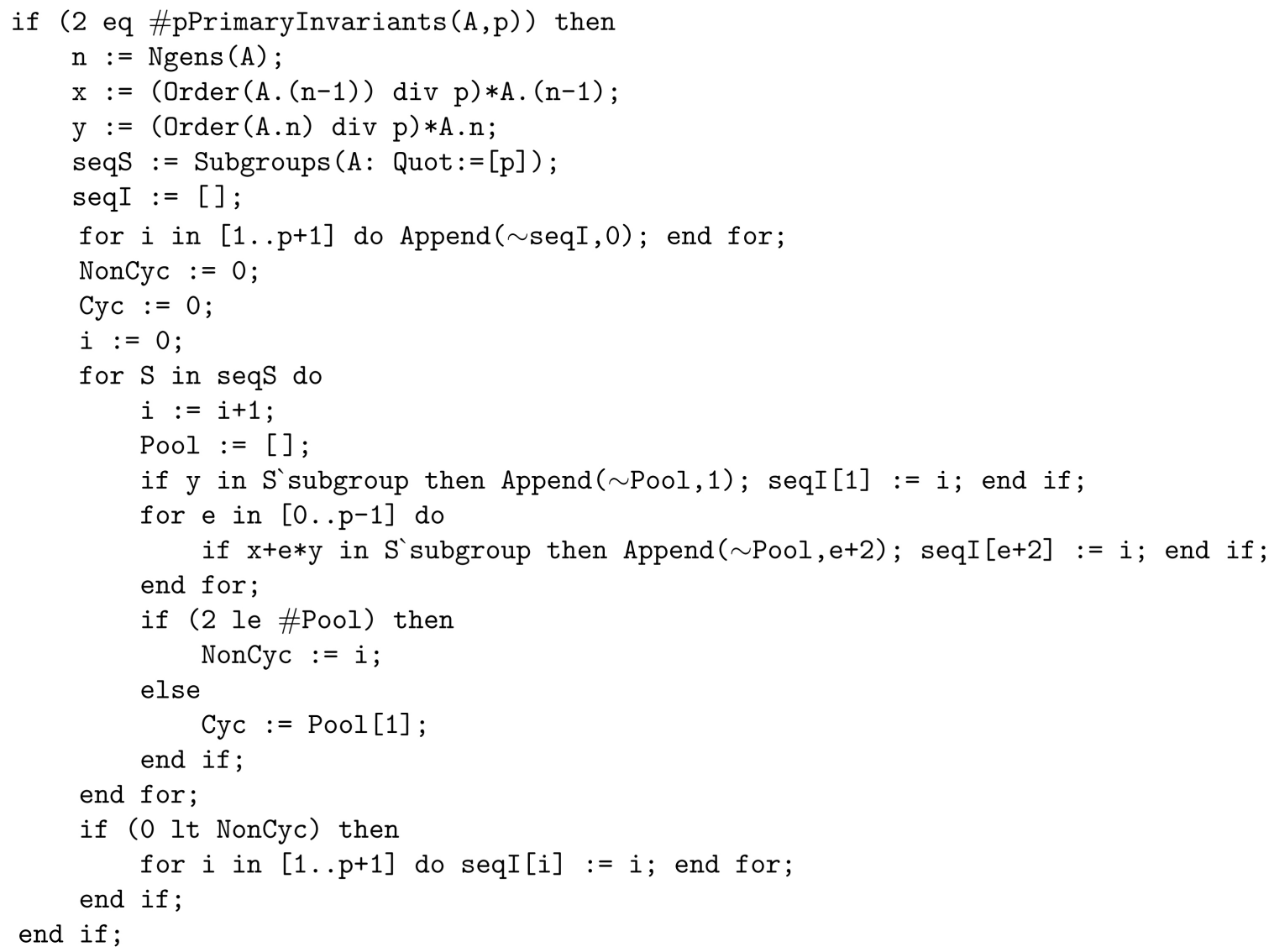

Output: Generators $x, y$ of the $p$-elementary subgroup $A_{p}$ of $A$, two indicators, NonCyc for one or more non-cyclic maximal subgroups of $\operatorname{Syl}_{p} A$, Cyc for one or more cyclic maximal subgroups of $\operatorname{Syl}_{p} A$, an ordered sequence seqS of the $p+1$ subgroups of $A$ of index $p$, and, if there are only cyclic maximal subgroups of $\operatorname{Syl}_{p} A$, an ordered sequence seqI of numerical identifiers for the elements $S$ of seqS.

Proof. This is precisely the implementation of the Propositions 2.1, 2.2 and 2.3 in MAGMA [8].

Remark 3.2. The modified statement seqS: =Subgroups(A: Quot:=[p,p]); yields the biggest subgroup of A of order coprime to $\mathrm{p}$, and can be used for constructing the Hilbert p-class field $\mathrm{F}_{p}^{1} K$ of the base field $\mathrm{K}$ in Algorithm 3.3, if the p-class group $\mathrm{Cl}_{p} K$ is of type $(p, p)$.

The class group $(C, m C)$ in the output of Algorithm 3.1 is used as input for Algorithm 3.2. The resulting sequence seqS of all subgroups of index $p$ in $C$, together with the pair $(C, m C)$, forms the input of Algorithm 3.3, which determines all unramified cyclic extensions $L_{i} \mid K$ of relative degree $p$ using the Artin correspondence as described by Fieker [14].

Algorithm 3.3. (Construction of all unramified cyclic extensions of degree $p$ ). 
Input: The class group $(C, m C)$ of a base field $K$ and the ordered sequence seq $S$ of all subgroups $S$ of index $p$ in $C$.

\section{Code:}

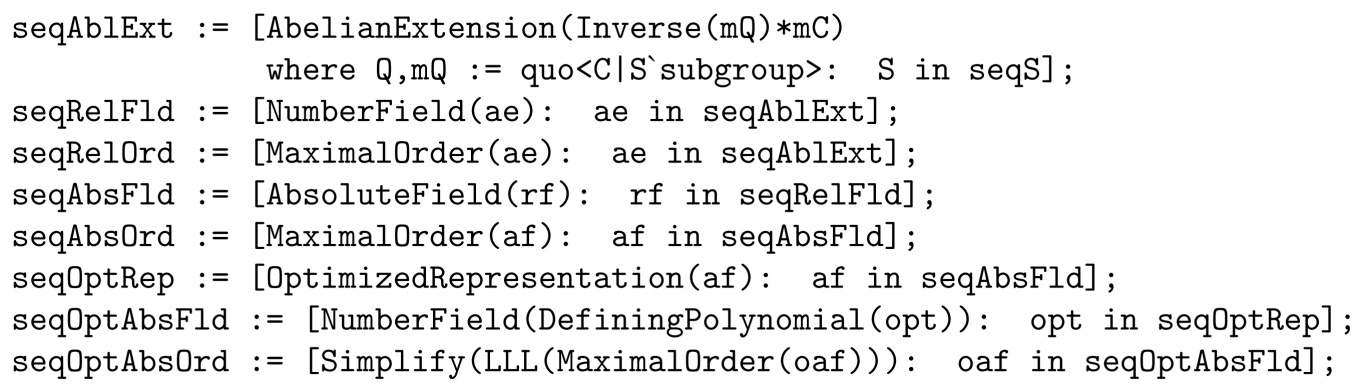

Output: Three ordered sequences, seqRelOrd of the relative maximal orders of $L_{i} \mid K$, seqAbsOrd of the corresponding absolute maximal orders of $L_{i} \mid \mathbb{Q}$, and seqOptAbsOrd of optimized representations for the latter.

Remark 3.3. Algorithm 3.3 is independent of the p-class rank $\varrho$ of the base field K. In order to obtain the adequate coercion of ideals, the sequence seqRelOrd must be used for computing the transfer kernel type $\varkappa(K)$ in Algorithm 3.4. The trailing three lines of Algorithm 3.3 are optional but highly recommended, since the size of all arithmetical invariants, such as polynomial coefficients, is reduced considerably. Either the sequence seqAbsOrd or rather the sequence seqOptAbsOrd should be used for calculating the transfer target type $\tau(K)$ in Algorithm 3.5.

Algorithm 3.4. (Transfer kernel type, $\varkappa(K))$.

Input: The prime number $p$, the ordered sequence seqRelOrd of the relative maximal orders of $L_{i} \mid K$, the class group mapping $m C$ of the base field $K$ with $p$-class rank $\varrho=2$, the generators $x, y$ of the $p$ elementary class group $E_{p}$ of $K$, and the ordered sequence seqI of numerical identifiers for the $p+1$ subgroups $S$ of index $p$ in the class group $C$ of $K$.

Code:

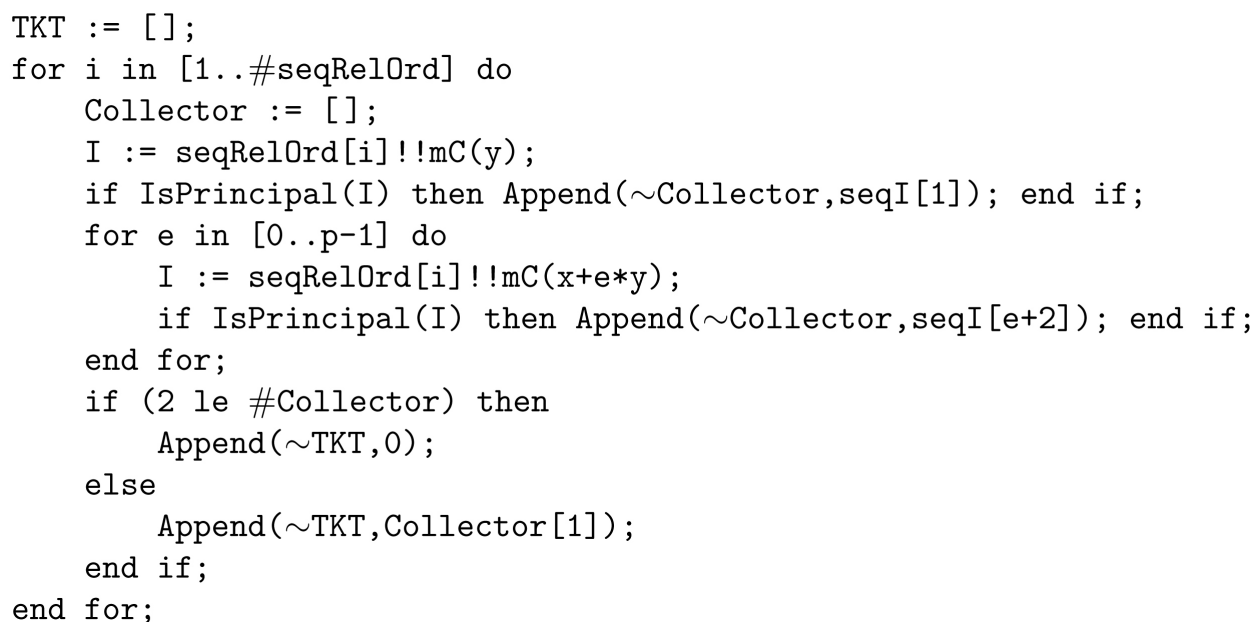

Output: The transfer kernel type TKT of $K$.

Remark 3.4. In 2012, Bembom investigated the 5-capitulation over complex quadratic fields $\mathrm{K}$ with 5-class group of type $(5,5)$ ([15] p. 129). However, his techniques were only able to distinguish between permutation types and nearly constant types, since he did not use the crucial sequence of numerical identifiers. We refined his results in ([16] §3.5, pp. 445-451) by determining the cycle decomposition and, in particular, the fixed points of the permutation types, which admitted the solution of an old problem by Taussky ([16] §3.5.2, p. 448).

Algorithm 3.5. (Transfer target type, $\tau(K)$ ). 
Input: The prime number $p$ and the ordered sequence seqOptAbsOrd of the optimized absolute maximal orders of $L_{i} \mid \mathbb{Q}$.

Code:

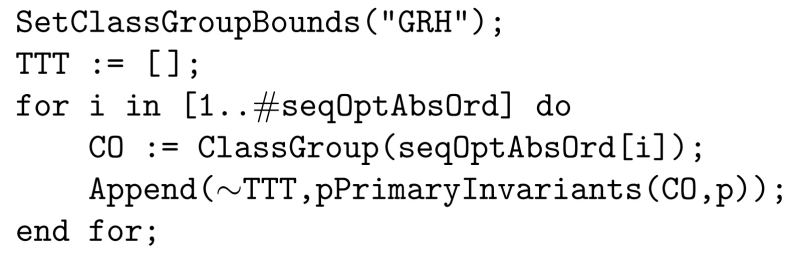

Output: The conditional transfer target type TTT of $K$, assuming the GRH.

With Algorithms 3.4 and 3.5 we are in the position to determine the Artin pattern $\mathrm{AP}(K)=(\tau(K), \varkappa(K))$ of the field $K$. For pointing out fixed points of the transfer kernel type $\varkappa(K)$ it is useful to define a corresponding weak TKT $\kappa=\kappa(K)$ which collects the Taussky conditions A, resp. B, of Theorem 2.1, for each extension $L_{i} \mid K$ :

$$
\kappa_{i}:= \begin{cases}\mathrm{A} & \text { if } \operatorname{ker} j_{L_{i} \mid K} \cap \operatorname{Norm}_{L_{i} \mid K} \mathrm{Cl}_{p} L_{i}>1, \\ \mathrm{~B} & \text { if ker } j_{L_{i} \mid K} \cap \operatorname{Norm}_{L_{i} \mid K} \mathrm{Cl}_{p} L_{i}=1 .\end{cases}
$$

Algorithm 3.6. (Weak transfer kernel type, $\kappa(K)$, containing Taussky's conditions A, resp. B) Input: The indicators NonCyc, Cyc, and the TKT.

\section{Code:}

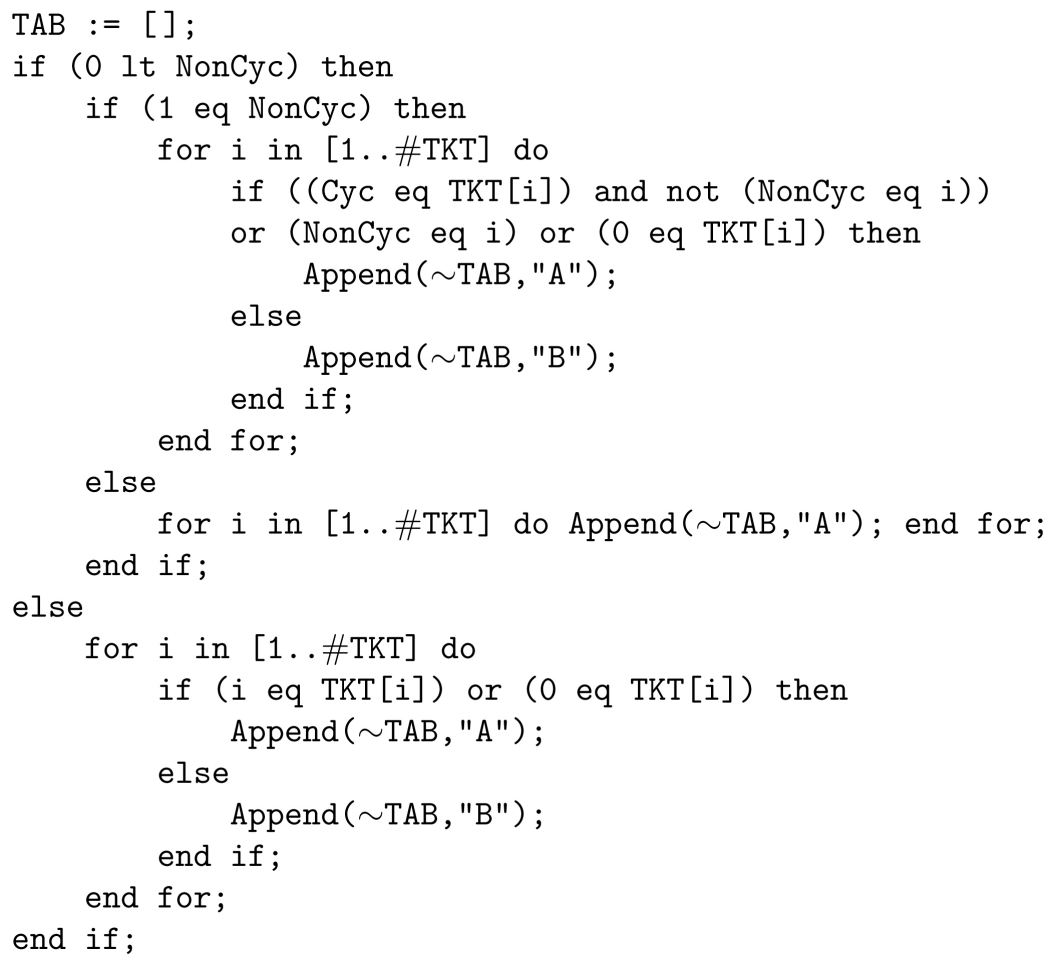

Output: The weak transfer kernel type TAB of $K$.

Proof. This is the implementation of Theorem 2.1 in MAGMA [8].

\section{Interpretation of Numerical Results}

By means of the algorithms in $\S 3$, we have computed the Artin pattern $\operatorname{AP}(K)=(\tau(K), \varkappa(K))$ of all 34,631 
real quadratic fields $K=\mathbb{Q}(\sqrt{d})$ with $\mathrm{Cl}_{3} K \simeq(3,3)$ in the range $0<d<10^{8}$ of fundamental discriminants. The results are presented in the following four tables, arranged by the coclass $\operatorname{cc}(\mathfrak{G})$ of the second 3-class group $\mathfrak{G}=\mathrm{G}_{3}^{2} K$. Each table gives the type designation, distinguishing ground states and excited states $\left(\uparrow, \uparrow^{2}, \ldots\right)$, the transfer kernel type $\varkappa=\varkappa(K)$, the transfer target type $\tau=\tau(K)$, the absolute frequency AF, the relative frequency RF, that is the percentage with respect to the total number of occurrences of the fixed coclass, and the minimal discriminant MD ([17] Dfn. 5.1). Additionally to this experimental information, we have identified the group $\mathfrak{G}$ by means of the strategy of pattern recognition via Artin transfers ([10] §4), and computed the factorized order of its automorphism group Aut($(\mathfrak{G})$ and its relation rank

$d_{2}(\mathfrak{G}):=\operatorname{dim}_{\mathbb{F}_{p}} H^{2}\left(\mathfrak{G}, \mathbb{F}_{p}\right)$. Groups are specified by their names in the SmallGroups Library [18] [19]. The nilpotency class $c=\operatorname{cl}(\mathfrak{G})$ and coclass $r=\operatorname{cc}(\mathfrak{G})$ were determined by means of ([20] Thm. 3.1, p. 290, and Thm. 3.2, p. 291), resp. ([17] Thm. 3.1).

\subsection{Groups $\mathfrak{G}$ of Coclass $\operatorname{cc}(\mathfrak{G})=1$}

The 31,088 fields whose second 3-class group $\mathfrak{G}$ is of maximal class, i.e. of $\operatorname{coclass} \operatorname{cc}(\mathfrak{G})=1$, constitute a contribution of $89.77 \%$, which is dominating by far. This confirms the tendency which was recogized for the restricted range $0<d<10^{7}$ already, where we had $\frac{2303}{2576} \approx 89.4 \%$ in ([3] Tbl. 2, p. 496) and ([9] Tbl. 6.1, p.

451). However, there is a slight increase of $0.37 \%$ for the relative frequency of $\operatorname{cc}(\mathfrak{G})=1$ in the extended range.

Theorem 4.1. (Coclass 1 ) The Hilbert 3 -class field tower of a real quadratic field $K$ whose second 3 -class group $\mathfrak{G}=\operatorname{Gal}\left(\mathrm{F}_{3}^{2} K \mid K\right)$ is of coclass $\operatorname{cc}(\mathfrak{G})=1$ has exact length $\ell_{3} K=2$, that is, the 3-class tower group $G=\operatorname{Gal}\left(\mathrm{F}_{3}^{\infty} K \mid K\right)$ is isomorphic to $\mathfrak{G}$, and $K<\mathrm{F}_{3}^{1} K<\mathrm{F}_{3}^{2} K=\mathrm{F}_{3}^{\infty} K$.

Proof. This is Theorem 5.3 in [17].

In Table 1, we denote two crucial mainline vertices of the unique coclass- 1 tree $\mathcal{T}^{1}\left(\left\langle 3^{2}, 2\right\rangle\right)$ by $M_{7}:=\left\langle 3^{7}, 386\right\rangle$ and $M_{9}:=M_{7}(-\# 1 ; 1)^{2}$, and we give the results for $\operatorname{cc}(\mathfrak{G})=1$.

The large scale separation of the types a.2 and a.3, resp. a. $2 \uparrow$ and a.3 $\uparrow$, in Table 1 became possible for the first time by our new algorithm. It refines the results in ([3] Tbl. 2, p. 496) and ([9] Tbl. 6.1, p. 451), and consequently also the frequency distribution in ([16] Fig. 3.2, p. 422).

Inspired by Boston, Bush and Hajir's theory of the statistical distribution of $p$-class tower groups of complex quadratic fields [21], we expect that, in Table 1 and in view of Theorem 4.1, the asymptotic limit of the relative frequency RF of realizations of a particular group $\mathfrak{G}=\mathrm{G}_{3}^{2} K \simeq G=\mathrm{G}_{3}^{\infty} K$ is proportional to the reciprocal of the order \#Aut(G) of its automorphism group. In particular, we state the following conjecture about three do-

Table 1. Statistics of 3-capitulation types $\varkappa=\varkappa(K)$ of fields $K$ with $\operatorname{cc}(\mathfrak{G})=1$.

\begin{tabular}{|c|c|c|c|c|c|c|c|c|}
\hline Type & $\varkappa$ & $\tau$ & $\mathrm{AF}$ & $\mathrm{RF}$ & MD & $\mathfrak{G}=G_{3}^{2}$ & \#Aut & $d_{2}$ \\
\hline а.1 & 0000 & $2^{2},\left(1^{2}\right)^{3}$ & 2180 & $7.01 \%$ & 62,501 & $\left\langle 3^{6}, 99 \ldots 101\right\rangle$ & $2^{1} 3^{8}$ & 3 \\
\hline a.2 & 1000 & $21,\left(1^{2}\right)^{3}$ & 7104 & $22.85 \%$ & 72,329 & $\left\langle 3^{4}, 10\right\rangle$ & $2^{1} 3^{5}$ & 3 \\
\hline a.3 & 2000 & $21,\left(1^{2}\right)^{3}$ & 10,514 & $33.82 \%$ & 32,009 & $\left\langle 3^{4}, 8\right\rangle$ & $2^{2} 3^{4}$ & 3 \\
\hline a. $3^{*}$ & 2000 & $1^{3},\left(1^{2}\right)^{3}$ & 10,244 & $32.95 \%$ & 142,097 & $\left\langle 3^{4}, 7\right\rangle$ & $2^{2} 3^{4}$ & 3 \\
\hline a. $1 \uparrow$ & 0000 & $3^{2},\left(1^{2}\right)^{3}$ & 58 & $0.19 \%$ & 2,905,160 & $M_{7}-\# 1 ; 5 \ldots 7$ & $2^{1} 3^{12}$ & 3 \\
\hline a. $2 \uparrow$ & 1000 & $32,\left(1^{2}\right)^{3}$ & 242 & $0.78 \%$ & 790,085 & $\left\langle 3^{6}, 96\right\rangle$ & $2^{1} 3^{9}$ & 3 \\
\hline а. $3 \uparrow$ & 2000 & $32,\left(1^{2}\right)^{3}$ & 713 & $2.29 \%$ & 494,236 & $\left\langle 3^{6}, 97 \mid 98\right\rangle$ & $2^{2} 3^{8}$ & 3 \\
\hline a. $1 \uparrow^{2}$ & 0000 & $4^{2},\left(1^{2}\right)^{3}$ & 3 & & $40,980,808$ & $M_{9}-\# 1 ; 5 \ldots 7$ & $2^{1} 3^{16}$ & 3 \\
\hline a. $2 \uparrow^{2}$ & 1000 & $43,\left(1^{2}\right)^{3}$ & 9 & $0.03 \%$ & $25,714,984$ & $M_{7}-\# 1 ; 2$ & $2^{1} 3^{13}$ & 3 \\
\hline a. $3 \uparrow^{2}$ & 2000 & $43,\left(1^{2}\right)^{3}$ & 20 & $0.06 \%$ & $10,200,108$ & $M_{7}-\# 1 ; 3 \mid 4$ & $2^{2} 3^{12}$ & 3 \\
\hline a. $2 \uparrow^{3}$ & 1000 & $54,\left(1^{2}\right)^{3}$ & 1 & & $37,304,664$ & $M_{9}-\# 1 ; 2$ & $2^{1} 3^{17}$ & 3 \\
\hline \multicolumn{3}{|c|}{ Total of $\operatorname{cc}(\mathfrak{G})=1$} & 31,088 & \multicolumn{5}{|c|}{$89.77 \%$ with respect to 34,631} \\
\hline
\end{tabular}


minating types, a. $3^{*}$, a.3 and a.2.

Conjecture 4.1. For a sufficiently extensive range $0<d<B$ of fundamental discriminants, both, the absolute and relative frequencies of realizations of the groups $\left\langle 3^{4}, 7\right\rangle,\left\langle 3^{4}, 8\right\rangle$ and $\left\langle 3^{4}, 10\right\rangle$, resp. $\left\langle 3^{6}, 97\right\rangle$, $\left\langle 3^{6}, 98\right\rangle$ and $\left\langle 3^{6}, 96\right\rangle$, as 3-class tower groups $G_{3}^{\infty} K=G_{3}^{2} K$ of real quadratic fields $K=\mathbb{Q}(\sqrt{d})$ satisfy the proportion $3: 3: 2$.

Proof. (Attempt of an explanation) A heuristic justification of the conjecture is given for the ground states by the relation for reciprocal orders

$$
\# \text { Aut }\left(\left\langle 3^{4}, 7\right\rangle\right)^{-1}=\# \operatorname{Aut}\left(\left\langle 3^{4}, 8\right\rangle\right)^{-1}=\frac{1}{2^{2} 3^{4}}=\frac{3}{2} \cdot \frac{1}{2^{1} 3^{5}}=\frac{3}{2} \cdot \# \operatorname{Aut}\left(\left\langle 3^{4}, 10\right\rangle\right)^{-1},
$$

which is nearly fulfilled by $10244 \approx 10514 \approx \frac{3}{2} \cdot 7104$, resp. $32.95 \% \approx 33.82 \% \approx \frac{3}{2} \cdot 22.85 \%$, for the bound $B=10^{8}$, and disproves our oversimplified conjectures at the end of ([10] Rmk. 5.2).

For the first excited states, we have the reciprocal orders

$$
\text { \#Aut }\left(\left\langle 3^{6}, 97\right\rangle\right)^{-1}=\# \operatorname{Aut}\left(\left\langle 3^{6}, 98\right\rangle\right)^{-1}=\frac{1}{2^{2} 3^{8}}=\frac{3}{2} \cdot \frac{1}{2^{1} 3^{9}}=\frac{3}{2} \cdot \# \operatorname{Aut}\left(\left\langle 3^{6}, 96\right\rangle\right)^{-1},
$$

but here no arithmetical invariants are known for distinguishing between $\left\langle 3^{6}, 97\right\rangle$ and $\left\langle 3^{6}, 98\right\rangle$, whence we have $713 \approx 3 \cdot 242$, resp. $2.29 \% \approx 3 \cdot 0.78 \%$, with cumulative factor $2 \cdot \frac{3}{2}=3$.

\subsection{Groups $\mathfrak{G}$ of Coclass $\operatorname{cc}(\mathfrak{G})=2$}

The 3328 fields whose second 3-class group $\mathfrak{G}$ is of second maximal class, i.e. of coclass $\operatorname{cc}(\mathfrak{G})=2$, constitute a moderate contribution of $9.61 \%$. The corresponding relative frequency for the restricted range $0<d<10^{7}$ is $\frac{260}{2576} \approx 10.1 \%$, which can be figured out from ([3] Tbl. 4-5, pp. 498-499) or, more easily, from

([9] Tbl. 6.3, Tbl. 6.5, Tbl. 6.7, pp. 452-453). So there is a slight decrease of $0.49 \%$ for the relative frequency of $\operatorname{cc}(\mathfrak{G})=2$ in the extended range.

Theorem 4.2. (Section D) The Hilbert 3-class field tower of a real quadratic field $K$ whose second 3 -class group $\mathfrak{G}=\operatorname{Gal}\left(\mathrm{F}_{3}^{2} K \mid K\right)$ is isomorphic to either of the two Schur $\sigma$-groups $\left\langle 3^{5}, 5\right\rangle$ or $\left\langle 3^{5}, 7\right\rangle$ has exact length $\ell_{3} K=2$, that is, the 3-class tower group $G=\mathrm{Gal}\left(\mathrm{F}_{3}^{\infty} K \mid K\right)$ is isomorphic to $\mathfrak{G}$, and $K<\mathrm{F}_{3}^{1} K<\mathrm{F}_{3}^{2} K=\mathrm{F}_{3}^{\infty} K$.

Proof. This statement has been proved by Scholz and Taussky in ([22] 3, p. 39). It has been confirmed with different techniques by Brink and Gold in ([23] Thm. 7, pp. 434-435), and by Heider and Schmithals in ([24] Lem. 5, p. 20). All three proofs were expressed for complex quadratic base fields $K$, but since the cover ([25] Dfn. 5.1, p. 30) of a Schur $\sigma$-group $\mathfrak{G}$ consists of a single element, $\operatorname{cov}(\mathfrak{G})=\{\mathfrak{G}\}$, the statement is actually valid for any algebraic number field $K$, in particular also for a real quadratic field $K$.

Table 2 shows the computational results for $\operatorname{cc}(\mathfrak{G})=2$, using the relative identifiers of the ANUPQ package [26] for groups $\mathfrak{G}$ of order $\# \mathfrak{G} \geq 3^{8}$, resp. $G$ of order $\# G \geq 3^{8}$. The possibilities for the 3-class tower group $G$ are complete for the TKTs c.18, c.21, E.6, E.8, E.9 and E.14, constituting the cover of the corresponding

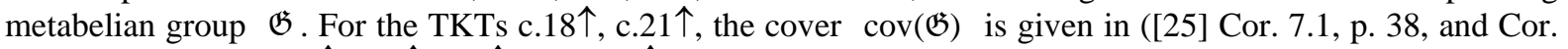

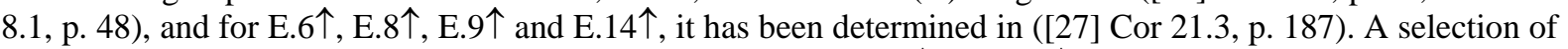
densely populated vertices is given for the sporadic TKTs G.19* and H.4*, according to ([17] Tbl. 4-5). We denote two important branch vertices of depth 1 by $N_{9, j}:=\left\langle 3^{7}, 303\right\rangle-\# 1 ; 1-\# 1 ; j$ for $j \in\{3,5\}$.

Whereas the sufficient criterion for $\ell_{3} K=2$ in Theorem 4.4 is known since 1934 already, the following statement of 2015 is brand-new and constitutes one of the few sufficient criteria for $\ell_{3} K=3$, that is, for the long desired three-stage class field towers [28].

Theorem 4.3. (Section $\mathrm{c}$ ) The Hilbert 3 -class field tower of a real quadratic field $K$ whose second 3 -class group $\mathfrak{G}=\operatorname{Gal}\left(\mathrm{F}_{3}^{2} K \mid K\right)$ is one of the six groups $\left\langle 3^{6}, 49\right\rangle,\left\langle 3^{6}, 54\right\rangle,\left\langle 3^{7}, 285\right\rangle-\# 1 ; 1,\left\langle 3^{7}, 303\right\rangle-\# 1 ; 1$, $\left\langle 3^{7}, 285\right\rangle(-\# 1 ; 1)^{3},\left\langle 3^{7}, 285\right\rangle(-\# 1 ; 1)^{3}$ has exact length $\ell_{3} K=3$, that is,

$K<\mathrm{F}_{3}^{1} K<\mathrm{F}_{3}^{2} K<\mathrm{F}_{3}^{3} K=\mathrm{F}_{3}^{\infty} K$.

Proof. This is the union of Thm. 7.1, Cor. 7.1, Cor 7.3, Thm 8.1, Cor 8.1, and Cor 8.3 in [25]. 
Table 2. Statistics of 3-capitulation types $\varkappa=\varkappa(K)$ of fields $K$ with $\operatorname{cc}(\mathfrak{G})=2$.

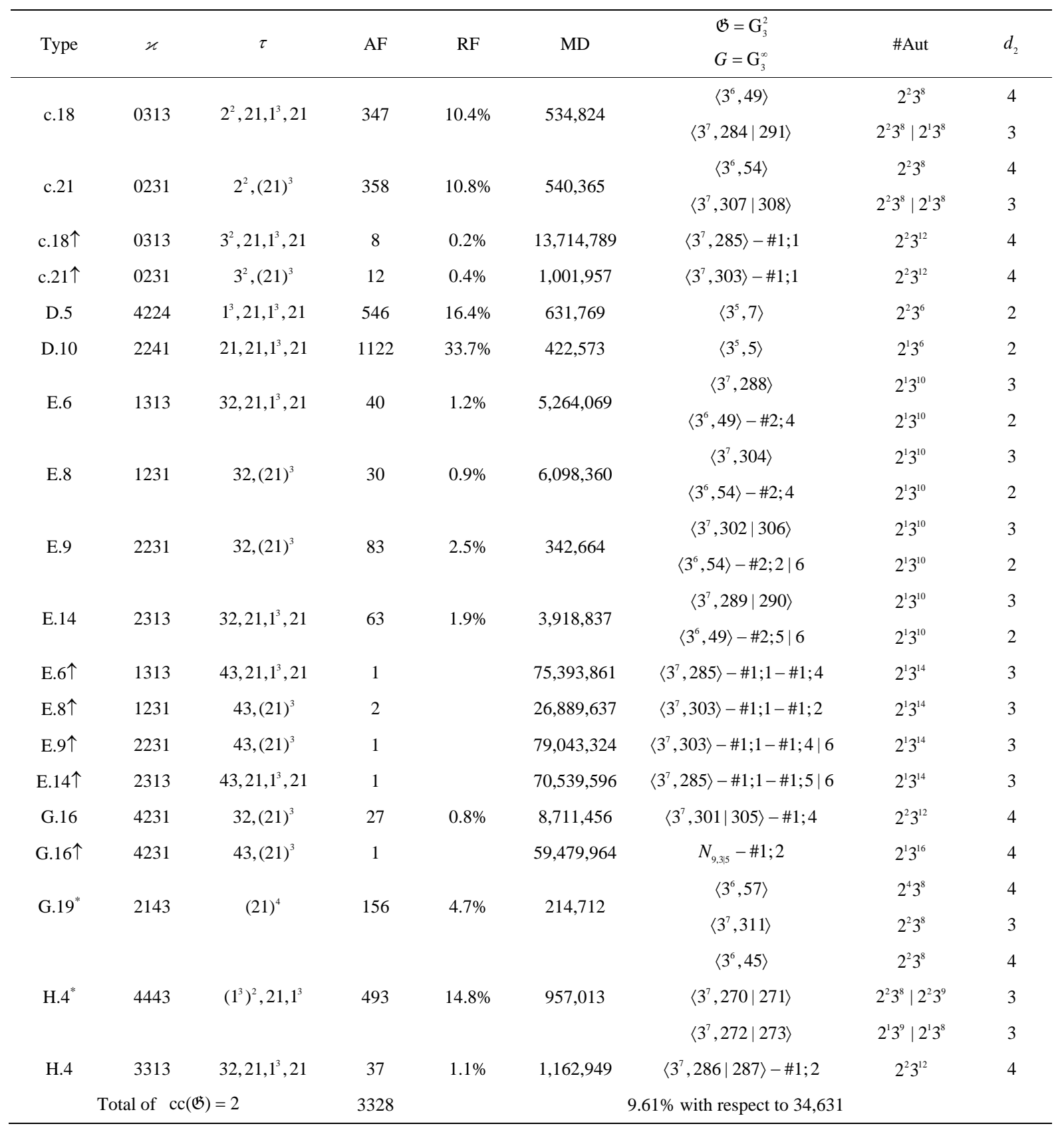

A sufficient criterion for $\ell_{3} K=3$ similar to Theorem 4.3 has been given in ([29] Thm. 6.1, pp. 751-752) for complex quadratic fields with TKTs in section E. Due to the relation rank $d_{2}$ of the involved groups, only a weaker statement is possible for real quadratic fields with such TKTs.

Theorem 4.4. (Section E) The Hilbert 3-class field tower of a real quadratic field $K$ whose second 3 -class group $\mathfrak{G}=\operatorname{Gal}\left(\mathrm{F}_{3}^{2} K \mid K\right)$ is one of the twelve groups $\left\langle 3^{7}, 288 \ldots 290\right\rangle,\left\langle 3^{7}, 302|304| 306\right\rangle$, $\left\langle 3^{7}, 285\right\rangle-\# 1 ; 1-\# 1 ; 4 \ldots 6,\left\langle 3^{7}, 303\right\rangle-\# 1 ; 1-\# 1 ; 2|4| 6$ has either length $\ell_{3} K=3$, that is, $K<\mathrm{F}_{3}^{1} K<\mathrm{F}_{3}^{2} K<\mathrm{F}_{3}^{3} K=\mathrm{F}_{3}^{\infty} K$, or length $\ell_{3} K=2$, that is, $K<\mathrm{F}_{3}^{1} K<\mathrm{F}_{3}^{2} K=\mathrm{F}_{3}^{\infty} K$.

Proof. This is the union of Thm. 4.1 and Thm. 4.2 in [17].

Example 4.1. That both cases $\ell_{3} K \in\{2,3\}$ occur with nearly equal frequency has been shown for the ground states in Thm. 5.5 and Thm. 5.6 of [17]. Due to our extended computations, we are now in the position to 
prove that the same is true for the first excited states. We have $\ell_{3} K=3$ for the two fields $K=\mathbb{Q}(\sqrt{d})$ with $d=70539596$, type E.14 $\uparrow$, and $d=75393861$, type E.6 $\uparrow$, but only $\ell_{3} K=2$ for the three fields with $d=79043324$, type E.9个, and $d \in\{26889637,98755469\}$, both of type E.8 ,

Recently, we have provided evidence of asymptotic frequency distributions for three-stage class field towers, similar to Conjecture 4.1 for two-stage towers.

Conjecture 4.2. For a sufficiently extensive range $0<d<B$ of fundamental discriminants, both, the absolute and relative frequencies of realizations of the groups $\left\langle 3^{7}, 284\right\rangle$ and $\left\langle 3^{7}, 291\right\rangle$, resp. $\left\langle 3^{7}, 307\right\rangle$ and $\left\langle 3^{7}, 308\right\rangle$ as 3-class tower groups $\mathrm{G}_{3}^{\infty} K=\mathrm{G}_{3}^{3} K$ of real quadratic fields $K=\mathbb{Q}(\sqrt{d})$ satisfy the proportion $1: 2$.

Proof. (Attempt of a heuristic justification of the conjecture)

For the first two groups, which form the cover of $\left\langle 3^{6}, 49\right\rangle$, we have the reciprocal order relation

$$
\text { \#Aut }\left(\left\langle 3^{7}, 291\right\rangle\right)^{-1}=\frac{1}{2^{1} 3^{8}}=2 \cdot \frac{1}{2^{2} 3^{8}}=2 \cdot \# A u t\left(\left\langle 3^{7}, 284\right\rangle\right)^{-1},
$$

which is nearly fulfilled by the statistical information $18 \approx 2 \cdot 10$, resp. $64 \% \approx 2 \cdot 36 \%$, given in ([25] Thm. 7.2, pp. 34-35) for $B=10^{7}$.

For the trailing two groups, which form the cover of $\left\langle 3^{6}, 54\right\rangle$, only arithmetical invariants of higher order are known for distinguishing between $\left\langle 3^{7}, 307\right\rangle$ and $\left\langle 3^{7}, 308\right\rangle$. It would have been too time consuming to compute these invariants for ([25] Thm. 8.2, p. 45).

Conjecture 4.3. For a sufficiently extensive range $0<d<B$ of fundamental discriminants, both, the absolute and relative frequencies of realizations of the groups $\left\langle 3^{7}, 270\right\rangle,\left\langle 3^{7}, 271\right\rangle,\left\langle 3^{7}, 272\right\rangle$ and $\left\langle 3^{7}, 273\right\rangle$ as 3-class tower groups $\mathrm{G}_{3}^{\infty} K=\mathrm{G}_{3}^{3} K$ of real quadratic fields $K=\mathbb{Q}(\sqrt{d})$ satisfy the proportion $3: 1: 2: 6$.

Proof. (Attempt of an explanation) All groups are contained in the cover of $\left\langle 3^{6}, 45\right\rangle$. We have the following relations between reciprocal orders

$$
\begin{aligned}
& \text { \#Aut }\left(\left\langle 3^{7}, 270\right\rangle\right)^{-1}=\frac{1}{2^{2} 3^{8}}=3 \cdot \frac{1}{2^{2} 3^{9}}=3 \cdot \text { \#Aut }\left(\left\langle 3^{7}, 271\right\rangle\right)^{-1}, \\
& \text { \#Aut }\left(\left\langle 3^{7}, 272\right\rangle\right)^{-1}=\frac{1}{2^{1} 3^{9}}=2 \cdot \frac{1}{2^{2} 3^{9}}=2 \cdot \text { \#Aut }\left(\left\langle 3^{7}, 271\right\rangle\right)^{-1}, \\
& \text { \#Aut }\left(\left\langle 3^{7}, 273\right\rangle\right)^{-1}=\frac{1}{2^{1} 3^{8}}=3 \cdot \frac{1}{2^{1} 3^{9}}=3 \cdot \text { \#Aut }\left(\left\langle 3^{7}, 272\right\rangle\right)^{-1} .
\end{aligned}
$$

Unfortunately, no arithmetical invariants are known for distinguishing between $\left\langle 3^{7}, 271\right\rangle$ and $\left\langle 3^{7}, 272\right\rangle$. Therefore, we must replace the two values in the middle of the proportion $3: 1: 2: 6$ by a cumulative value $3: 3: 6$, resp. $1: 1: 2$. The resulting proportion is fulfilled approximately by the statistical information $2 \cdot 5 \approx 2 \cdot 8 \approx 11$, resp. $2 \cdot 19 \% \approx 2 \cdot 29 \% \approx 41 \%$, given in ([17] Thm. 5.7) for $B=10^{7}$. However, a total of 24 individuals cannot be viewed as a statistical ensemble yet.

\subsection{Groups $\mathfrak{G}$ of Coclass $\operatorname{cc}(\mathfrak{G})=3$}

There are 190 fields whose second 3-class group $\mathfrak{G}$ is of coclass $\operatorname{cc}(\mathfrak{G})=3$. They constitute a very small contribution of $0.55 \%$. The corresponding relative frequency for the restricted range $0<d<10^{7}$ is $\frac{10}{2576} \approx 0.4 \%$, which can be figured out from ([3] Tbl. 5, p. 499) or, more easily, from ([9] Tbl. 6.2, p. 451). Thus, there is a slight increase of $0.15 \%$ for the relative frequency of $\operatorname{cc}(\mathfrak{G})=3$ in the extended range.

For the groups $\mathfrak{G}$ of coclass $\operatorname{cc}(\mathfrak{G}) \geq 3$, the problem of determining the corresponding 3-class tower group $G$ is considerably harder than for $\operatorname{cc}(\mathfrak{G}) \leq 2$, and up to now it is still open.

In Table 3, we denote two important mainline vertices of the coclass-2 tree $\mathcal{T}^{2}\left(\left\langle 3^{7}, 64\right\rangle\right)$ by $P_{7}:=\left\langle 3^{7}, 64\right\rangle$ and $P_{9}:=P_{7}-\# 1 ; 3-\# 1 ; 1$, and we give the statistics for $\operatorname{cc}(\mathfrak{G})=3$.

\subsection{Groups $\mathfrak{G}$ of Coclass $\operatorname{cc}(\mathfrak{G})=4$}

We only have 25 fields whose second 3-class group $\mathfrak{G}$ is of coclass $\operatorname{cc}(\mathfrak{G})=4$. They constitute a negligible contribution of $0.07 \%$. The corresponding relative frequency for the restricted range $0<d<10^{7}$ is 
$\frac{3}{2576} \approx 0.1 \%$, which can be seen in ([9] Tbl. 6.9, p. 454). So there is a slight decrease of $0.03 \%$ for the relative frequency of $\operatorname{cc}(\mathfrak{G})=4$ in the extended range.

In Table 4, we denote some crucial mainline vertices of coclass-4 trees $\mathcal{T}^{4}\left(S_{9, j}\right)$ by

$$
\begin{gathered}
S_{9, j}:=\left\langle 3^{7}, 64\right\rangle-\# 2 ; j \text { and } S_{10,39}:=S_{9,39}-\# 1 ; 7, \quad S_{10,44}:=S_{9,44}-\# 1 ; 1, \quad S_{10,54}:=S_{9,54}-\# 1 ; 8, \\
S_{10,57}:=S_{9,57}-\# 1 ; 1, \quad S_{10,59}:=S_{9,59}-\# 1 ; 6,
\end{gathered}
$$

a sporadic vertex by $T_{9}:=\left\langle 3^{7}, 64\right\rangle-\# 2 ; 34$, and we give the computational results for $\operatorname{cc}(\mathfrak{G})=4$.

For the essential difference between the location of the groups $\mathfrak{G}$ as vertices of coclass trees for the types d.25 ${ }^{*}$ and d.25, see ([30] Thm. 3.3-3.4 and Exm. 3.1, pp. 490-492).

The single occurrence of type $\mathrm{H} .4$ belongs to the irregular variant (i), where $\mathrm{Cl}_{3} \mathrm{~F}_{3}^{1} K \simeq(9,9,9,9)$. This is

\begin{tabular}{|c|c|c|c|c|c|c|c|c|}
\hline Type & $\varkappa$ & $\tau$ & $\mathrm{AF}$ & $\mathrm{RF}$ & MD & $\mathfrak{G}=\mathrm{G}_{3}^{2}$ & \#Aut & $d_{2}$ \\
\hline b.10 & 0043 & $\left(2^{2}\right)^{2},\left(1^{3}\right)^{2}$ & 95 & $50.0 \%$ & 710,652 & $P_{7}-\# 1 ; 21 \ldots 26$ & $2^{2} 3^{12} \mid 2^{1} 3^{12}$ & 5 \\
\hline b. $10 \uparrow$ & 0043 & $3^{2}, 2^{2},\left(1^{3}\right)^{2}$ & 6 & $3.2 \%$ & $17,802,872$ & $P_{9}-\# 1 ; 21 \ldots 29$ & $2^{1} 3^{16}$ & 5 \\
\hline d.19 & 4043 & $32,2^{2},\left(1^{3}\right)^{2}$ & 49 & $26.0 \%$ & $2,328,721$ & $P_{7}-\# 1 ; 4 \mid 5$ & $2^{1} 3^{12}$ & 5 \\
\hline d.23 & 1043 & $32,2^{2},\left(1^{3}\right)^{2}$ & 16 & $8.4 \%$ & $1,535,117$ & $P_{7}-\# 1 ; 6$ & $2^{1} 3^{12}$ & 5 \\
\hline d.25 & 2043 & $32,2^{2},\left(1^{3}\right)^{2}$ & 22 & $12.0 \%$ & $15,230,168$ & $P_{7}-\# 1 ; 7 \mid 8$ & $2^{2} 3^{12}$ & 5 \\
\hline d. $19 \uparrow$ & 4043 & $43,2^{2},\left(1^{3}\right)^{2}$ & 1 & & $27,970,737$ & $P_{9}-\# 1 ; 2 \mid 3$ & $2^{1} 3^{16}$ & 5 \\
\hline d. $23 \uparrow$ & 1043 & $43,2^{2},\left(1^{3}\right)^{2}$ & 1 & & $87,303,181$ & $P_{9}-\# 1 ; 4$ & $2^{1} 3^{16}$ & 5 \\
\hline \multicolumn{3}{|c|}{ Total of $\operatorname{cc}(\mathfrak{G})=3$} & 190 & \multicolumn{5}{|c|}{$0.55 \%$ with respect to 34,631} \\
\hline
\end{tabular}

Table 3. Statistics of 3-capitulation types $\varkappa=\varkappa(K)$ of fields $K$ with $\operatorname{cc}(\mathfrak{G})=3$.

\begin{tabular}{|c|c|c|c|c|c|c|c|c|}
\hline Type & $\varkappa$ & $\tau$ & $\mathrm{AF}$ & $\mathrm{RF}$ & MD & $\mathfrak{G}=\mathrm{G}_{3}^{2}$ & \#Aut & $d_{2}$ \\
\hline d. $25^{*}$ & 0143 & $3^{2}, 32,\left(1^{3}\right)^{2}$ & 4 & $16 \%$ & $8,491,713$ & $S_{10,5759}$ & $2^{2} 3^{16}$ & 5 \\
\hline \multirow[t]{2}{*}{ F.7 } & 3443 & $(32)^{2},\left(1^{3}\right)^{2}$ & 3 & $12 \%$ & $10,165,597$ & $P_{7}-\# 2 ; 55$ & $2^{1} 3^{14}$ & 4 \\
\hline & & & & & & $P_{7}-\# 2 ; 56 \mid 58$ & $2^{2} 3^{14}$ & 4 \\
\hline F.11 & 1143 & $(32)^{2},\left(1^{3}\right)^{2}$ & 3 & $12 \%$ & $66,615,244$ & $P_{7}-\# 2 ; 36 \mid 38$ & $2^{1} 3^{14}$ & 4 \\
\hline F.12 & 1343 & $(32)^{2},\left(1^{3}\right)^{2}$ & 6 & $24 \%$ & $22,937,941$ & $P_{7}-\# 2 ; 43|46| 51 \mid 53$ & $2^{1} 3^{14}$ & 4 \\
\hline F.13 & 3143 & $(32)^{2},\left(1^{3}\right)^{2}$ & 5 & $20 \%$ & $8,321,505$ & $P_{7}-\# 2 ; 41|47| 50 \mid 52$ & $2^{1} 3^{14}$ & 4 \\
\hline F.7个 & 3443 & $43,32,\left(1^{3}\right)^{2}$ & 1 & & $24,138,593$ & $S_{10,3914}-\# 1 ; 5 \mid 6$ & $2^{1} 3^{18}$ & 4 \\
\hline \multirow[t]{2}{*}{ F.12个 } & 1343 & $43,32,\left(1^{3}\right)^{2}$ & 1 & & $86,865,820$ & $S_{10,39}-\# 1 ; 2\left|9, \quad S_{10,44}-\# 1 ; 3\right| 8$ & $2^{1} 3^{18}$ & 4 \\
\hline & & & & & & $S_{10,54}-\# 1 ; 2|4| 6 \mid 8$ & $2^{1} 3^{18}$ & 4 \\
\hline \multirow[t]{2}{*}{ F.13个 } & 3143 & $43,32,\left(1^{3}\right)^{2}$ & 1 & & $8,127,208$ & $S_{10,39}-\# 1 ; 3\left|8, \quad S_{10,44}-\# 1 ; 2\right| 9$ & $2^{1} 3^{18}$ & 4 \\
\hline & & & & & & $S_{10,57}-\# 1 ; 2\left|4, \quad S_{10,59}-\# 1 ; 3\right| 4$ & $2^{1} 3^{18}$ & 4 \\
\hline \multirow[t]{2}{*}{ H.4i } & 4443 & $43,32,\left(1^{3}\right)^{2}$ & 1 & & $54,313,357$ & $T_{9}-\# 1 ; 7$ & $2^{1} 3^{16}$ & 4 \\
\hline & \multicolumn{2}{|c|}{ Total of $\operatorname{cc}(\mathfrak{G})=4$} & 25 & \multicolumn{5}{|c|}{$0.07 \%$ with respect to 34,631} \\
\hline
\end{tabular}

Table 4. Statistics of 3-capitulation types $\varkappa=\varkappa(K)$ of fields $K$ with $\operatorname{cc}(\mathfrak{G})=4$. 
explained in ([3] p. 498) and ([9] pp. 454-455). It is the only case in Table 4 where $\mathfrak{G}$ is determined uniquely.

\section{Acknowledgements}

The author gratefully acknowledges that his research is supported by the Austrian Science Fund (FWF): P 26008-N25.

\section{References}

[1] Mayer, D.C. (1992) Multiplicities of Dihedral Discriminants. Mathematics of Computation, 58, 831-847 and S55-S58.

[2] Mayer, D.C. (1990) Principalization in Complex $S_{3}$-Fields. Congressus Numerantium, 80, 73-87. Proceedings of the 12th Manitoba Conference on Numerical Mathematics and Computing, Winnipeg.

[3] Mayer, D.C. (2012) The Second p-Class Group of a Number Field. International Journal of Number Theory, 8, 471505.

[4] Herbrand, J. (1932) Sur les théorèmes du genre principal et des idéaux principaux. Abhandlungen aus dem Mathematischen Seminar der Universität Hamburg, 9, 84-92. http://dx.doi.org/10.1007/BF02940630

[5] Hilbert, D. (1897) Die Theorie der algebraischen Zahlkörper. Jber. der DMV, 4, 175-546.

[6] Chang, S.M. (1977) Capitulation Problems in Algebraic Number Fields. Ph.D. Thesis, University of Toronto, Toronto.

[7] Chang, S.M. and Foote, R. (1980) Capitulation in Class Field Extensions of Type ( $p, p)$. Canadian Journal of Mathematics, 32, 1229-1243.

[8] The MAGMA Group (2016) MAGMA Computational Algebra System. Version 2.22-1, Sydney. http://magma.maths.usyd.edu.au

[9] Mayer, D.C. (2014) Principalization Algorithm via Class Group Structure. Journal de Théorie des Nombres de Bordeaux, 26, 415-464.

[10] Mayer, D.C. (2016) Three-Stage Towers of 5-Class Fields. Journal of Number Theory. arXiv:1604.06930v1 [math.NT]

[11] Mayer, D.C. (2016) Artin Transfer Patterns on Descendant Trees of Finite p-Groups. Advances in Pure Mathematics, 6, 66-104. Special Issue on Group Theory Research, January 2016.

[12] Bosma, W., Cannon, J. and Playoust, C. (1997) The Magma Algebra System. I. The User Language. Journal of Symbolic Computation, 24, 235-265. http://dx.doi.org/10.1006/jsco.1996.0125

[13] Bosma, W., Cannon, J.J., Fieker, C. and Steels, A. (2016) Handbook of Magma Functions. Edition 2.22, Sydney.

[14] Fieker, C. (2001) Computing Class Fields via the Artin Map. Mathematics of Computation, 70, 1293-1303.

[15] Bembom, T. (2012) The Capitulation Problem in Class Field Theory. Dissertation, Georg-August-Universität Göttingen, 2012.

[16] Mayer, D.C. (2013) The Distribution of Second p-Class Groups on Coclass Graphs. Journal de Théorie des Nombres de Bordeaux, 25, 401-456.

[17] Mayer, D.C. (2016) Index-p abelianization Data of p-Class Tower Groups, II. Journal de Théorie des Nombres de Bordeaux. arXiv:1601.00179v1 [math.NT]

[18] Besche, H.U., Eick, B. andO’Brien, E.A. (2002) A Millennium Project: constructing Small Groups. International Journal of Algebra and Computation, 12, 623-644. http://dx.doi.org/10.1142/S0218196702001115

[19] Besche, H.U., Eick, B. and O’Brien, E.A. (2005) The Small Groups Library-A Library of Groups of Small Order, 2005, an Accepted and Refereed GAP Package. MAGMA.

[20] Mayer, D.C. (2015) Index-p Abelianization Data of p-Class Tower Groups. Advances in Pure Mathematics, 5, $286-313$. Special Issue on Number Theory and Cryptography, April 2015.

[21] Boston, N., Bush, M.R. and Hajir, F. (2014) Heuristics for p-Class Towers of Imaginary Quadratic Fields. Mathematische Annalen. arXiv:1111.4679v2 [math.NT]

[22] Scholz, A. and Taussky, O. (1934) Die Hauptideale der kubischen Klassenkörper imaginär quadratischer Zahlkörper: ihre rechnerische Bestimmung und ihr Einfluß auf den Klassenkörperturm. Journal für die Reine und Angewandte Mathematik, 171, 19-41.

[23] Brink, J.R. and Gold, R. (1987) Class Field towers of Imaginary Quadratic Fields. Manuscripta Mathematic, 57, 425450.

[24] Heider, F.-P. and Schmithals, B. (1982) Zur Kapitulation der Idealklassen in unverzweigten primzyklischen Erweiterungen. Journal für die reine und Angewandte Mathematik, 336, 1-25. 
[25] Mayer, D.C. (2015) New Number Fields with Known p-Class Tower. Tatra Mountains Mathematical Publications, 64, 21-57.

[26] Gamble, G, Nickel, W. and O’Brien, E.A. (2006) ANU p-Quotient-p-Quotient and p-Group Generation Algorithms, 2006, an Accepted GAP Package. MAGMA.

[27] Mayer, D.C. (2015) Periodic Bifurcations in Descendant Trees of Finite p-Groups. Advances in Pure Mathematics, 5, 162-195. Special Issue on Group Theory, March 2015.

[28] Bush, M.R. and Mayer, D.C. (2015) 3-Class Field Towers of Exact Length 3. Journal of Number Theory, 147, 766777. http://dx.doi.org/10.1016/j.jnt.2014.08.010

[29] Mayer, D.C. (2015) Periodic Sequences of P-Class Tower Groups. Journal of Applied Mathematics and Physics, 3, 746-756.

[30] Mayer, D.C. (2012) Transfers of Metabelian p-Groups. Monatshefte für Mathematik, 166, 467-495.

\section{Submit or recommend next manuscript to SCIRP and we will provide best service for you:}

Accepting pre-submission inquiries through Email, Facebook, LinkedIn, Twitter, etc.

A wide selection of journals (inclusive of 9 subjects, more than 200 journals)

Providing 24-hour high-quality service

User-friendly online submission system

Fair and swift peer-review system

Efficient typesetting and proofreading procedure

Display of the result of downloads and visits, as well as the number of cited articles

Maximum dissemination of your research work

Submit your manuscript at: http://papersubmission.scirp.org/ 